\title{
Expression of Glut-1 is a prognostic marker for oral squamous cell carcinoma patients
}

\author{
A.W.ECKERT ${ }^{1}$, M.H.W. LAUTNER ${ }^{1}$, H. TAUBERT ${ }^{1}$, J. SCHUBERT ${ }^{1}$ and U. BILKENROTH ${ }^{2}$ \\ ${ }^{1}$ Department of Oral and Maxillofacial Plastic Surgery, Martin-Luther-University Halle-Wittenberg, Ernst-Grube Str. 40, \\ D-06097 Halle/Saale; ${ }^{2}$ Institute of Pathology, Hospital of Eisleben, Hohetorstr. 25, D-06295 Eisleben, Germany
}

Received May 23, 2008; Accepted June 26, 2008

DOI: 10.3892/or_00000156

\begin{abstract}
Oral squamous cell carcinoma (OSCC) is among the tenth most common human cancers worldwide with evidence of an increase in incidence rate and mortality. Despite advances in treatment modalities, the prognosis of this cancer is still very poor and has not changed over the past two decades. This study is based on samples collected from 42 patients with a primary OSCC. Immunohistochemical staining for Glut-1 was carried out and compared with the clinicopathological data. Thirty-two patients showed in their tumors a weak or undetectable Glut-1 expression, whereas in tumors of 10 patients a moderate to strong Glut- 1 expression was detected. In multivariate Cox's regression hazard analysis, patients whose tumors had a moderate to strong Glut-1 expression possessed a 4.9-fold increased risk of tumorrelated death compared to the other patients. Our results suggest that Glut-1 expression is an independent prognostic marker for routine assessment of OSCC.
\end{abstract}

\section{Introduction}

Oral squamous cell carcinoma (OSCC) is one of the ten most common human cancers worldwide (1). The overall 5-year survival time of OSCC is only $40 \%$ (2-4). Despite advances in surgical techniques over the past two decades including microvascular flap-based intraoral reconstruction, no significant increase in overall survival and disease-free survival has been observed (2). Various markers including p53 (5), were investigated for their correlation with 5-year survival of patients (6). The most promising markers are vascular endothelial growth factor (VEGF) and the human glucose transporter (Glut-1) (7-9). Accelerating malignant tumors require blood vessel formation (angiogenesis) for an

Correspondence to: Dr Alexander W. Eckert, Department of Oral and Maxillofacial Plastic Surgery, Martin-Luther-University Halle-Wittenberg, Ernst-Grube-Strasse 40, D-06097 Halle/Saale, Germany

E-mail: aw.eckert.wissenschaft@web.de

Key words: immunohistochemistry, oral squamous cell carcinoma, prognosis, tumor hypoxia, Glut-1 increased rate of respiration and nutrients to operate. In hypoxic tumor cells, this upregulation of glycolytic metabolism helps to maintain the increased energy requirements of rapidly proliferating cells. Detection of these metabolic changes may be used to provide diagnostic and therapeutic information. Recently, the hypoxia-induced factor (HIF1) regulated protein Glut-1 was validated as an intrinsic marker of tumor hypoxia (10). Therefore, the study goal was first to evaluate the immunohistochemical expression of Glut-1 in a series of OSCC and to correlate these results with the clinical courses. Based on these findings, the prognostic impact of Glut-1 expression for 5-year overall survival and disease-free survival of OSCC patients was evaluated.

\section{Materials and methods}

Patients. The records of 42 patients surgically treated with or without radiotherapy were reviewed retrospectively. The patients were investigated with regard to localization of the primary tumor, the extent of lymph node metastasis and the operative strategy (Table I). Their 5-year survival rate was estimated by Kaplan-Meier analysis and was investigated in relation to age, gender, tumor site, tumor staging and tumor differentiation, as described in the literature $(11,12)$.

Immunohistochemical staining for Glut-1. Immunohistochemistry was performed on paraffin sections of 42 OSCC mounted on glass slides. Surgical specimens were fixed in $10 \%$ neutral-buffered formalin ( $\mathrm{pH} \mathrm{7.4)}$ at $4^{\circ} \mathrm{C}$ for $24 \mathrm{~h}$ and embedded in paraffin. Four- $\mu \mathrm{m}$ thick tissue sections were used and dewaxed in xylene and rehydrated through decreasing concentrations of ethanol and water. Immunohistochemical staining for Glut-1 expression was carried out using a standard immunoperoxidase technique (ABC-method). All slides were warmed up for $30 \mathrm{~min}$ to a temperature of $60^{\circ} \mathrm{C}$ for dewaxing, boiled in $10 \mathrm{mM}$ citrate buffer ( $\mathrm{pH} \mathrm{6.0)}$ by microwave treatment $(600 \mathrm{~W})$ for 20 min for demasking the antigens. All stainings were performed at coverslides in combination with the prepared slides; human placenta as positive control and one negative control were included. Endogenous peroxidase activity was blocked by immersion of slides in methanol with $0.03 \%$ hydrogen peroxide for $12 \mathrm{~min}$. The primary antibody was incubated for $30 \mathrm{~min}$ at a temperature of $37^{\circ} \mathrm{C}$ (dilution 1:200, Acris antibodies, Germany). After washing with PBS-buffer, the secondary antibody was applied at room 
Table I. Patient characteristics and Glut-1 expression in 42 OSCC specimens.

\begin{tabular}{|c|c|c|c|c|c|}
\hline \multirow[b]{2}{*}{ Parameter } & \multirow[b]{2}{*}{ Number $(\%)$} & \multicolumn{4}{|c|}{ Staining intensity } \\
\hline & & I & II & III & IV \\
\hline \multicolumn{6}{|l|}{ Age (years) } \\
\hline Mean (range) & $61(26-83)$ & & & & \\
\hline \multicolumn{6}{|l|}{ Gender } \\
\hline Male & $33(78.6)$ & 21 & 5 & 5 & 2 \\
\hline Female & $9(21.4)$ & 4 & 2 & 3 & 0 \\
\hline \multicolumn{6}{|c|}{ Localization of primary tumor } \\
\hline Tongue & $18(4.9)$ & 13 & 1 & 3 & 1 \\
\hline Floor of the mouth & $13(30.9)$ & 7 & 2 & 3 & 1 \\
\hline Buccal mucosa & $1(2.4)$ & 0 & 0 & 1 & 0 \\
\hline Tretromolar area & $6(7.0)$ & 3 & 2 & 1 & 0 \\
\hline Maxilla & $4(9.5)$ & 0 & 2 & 2 & 0 \\
\hline \multicolumn{6}{|l|}{ Tumor size } \\
\hline $\mathrm{T} 1$ & $10(23.8)$ & 8 & 1 & 1 & 0 \\
\hline $\mathrm{T} 2$ & $20(47.6)$ & 12 & 4 & 3 & 1 \\
\hline $\mathrm{T} 3$ & $4(9.5)$ & 3 & 0 & 0 & 1 \\
\hline $\mathrm{T} 4$ & $8(19.0)$ & 2 & 2 & 4 & 0 \\
\hline \multicolumn{6}{|l|}{ Lymph node status } \\
\hline No & $19(45.2)$ & 12 & 2 & 5 & 0 \\
\hline N1 & $15(35.7)$ & 10 & 1 & 3 & 1 \\
\hline $\mathrm{N} 2$ & $8(19.0)$ & 3 & 4 & 0 & 1 \\
\hline N3 & 0 & 0 & 0 & 0 & 0 \\
\hline \multicolumn{6}{|l|}{ Tumor stage } \\
\hline I & $8(19.0)$ & 7 & 0 & 1 & 0 \\
\hline II & $14(33.3)$ & 11 & 1 & 2 & 0 \\
\hline III & $4(9.5)$ & 2 & 0 & 1 & 1 \\
\hline IV & $16(38.1)$ & 5 & 6 & 4 & 1 \\
\hline \multicolumn{6}{|l|}{ Histological grade } \\
\hline Poorly & $12(28.6)$ & 7 & 1 & 2 & 0 \\
\hline Moderately & $20(47.6)$ & 12 & 3 & 4 & 1 \\
\hline Well & $10(23.8)$ & 6 & 3 & 2 & 1 \\
\hline
\end{tabular}

Staining intensity I, IRS 0-2 (negative); II, IRS 3-4 (weak); III, IRS, 6-8 (moderate) and IV, IRS 9-12 (strong).

temperature for $12 \mathrm{~min}$. The staining for Glut-1 was visualized with the chromogen kit (Zymed). The specimens were counterstained with hemalum (Midas E).

The stained specimens were viewed at an objective magnification of $\mathrm{x} 100$ and $\mathrm{x} 200$ by three investigators (U.B., A.W.E. and M.L.). The staining intensity was calculated following the score system by Remmele (13): 0-2, no staining; 3-4, weak staining; 6-8, moderate staining and 9-12, strong staining.

Statistical analysis. The immunohistochemical results were correlated with the clinical data in statistical tests $\left(\chi^{2}\right.$ test, Kaplan-Meier analysis, Log-rank test and Cox's regression hazard analysis (14).

\section{Results}

This study was performed on 42 patients with an OSCC diagnosed between 1993 and 1998. There were 31 women $(29.2 \%)$ and $75(70.8 \%)$ men, age range from 26 to 83 years. The main clinical and pathological characteristics of the selected 42 patients are described in Table I. The (5-year) overall survival of the patients determined in Kaplan-Meier analysis was $62.6 \%$.

Staining intensities of the tumors were in 10 cases moderate to strong and in 32 cases negative to weak (Fig. 1). Expression of Glut-1 was not correlated to tumor grade, tumor stage and lymph node metastasis, but a weak association to tumor size ( $\chi^{2}$ test, $\left.\mathrm{P}=0.086\right)$ was determined. Then, we 
A

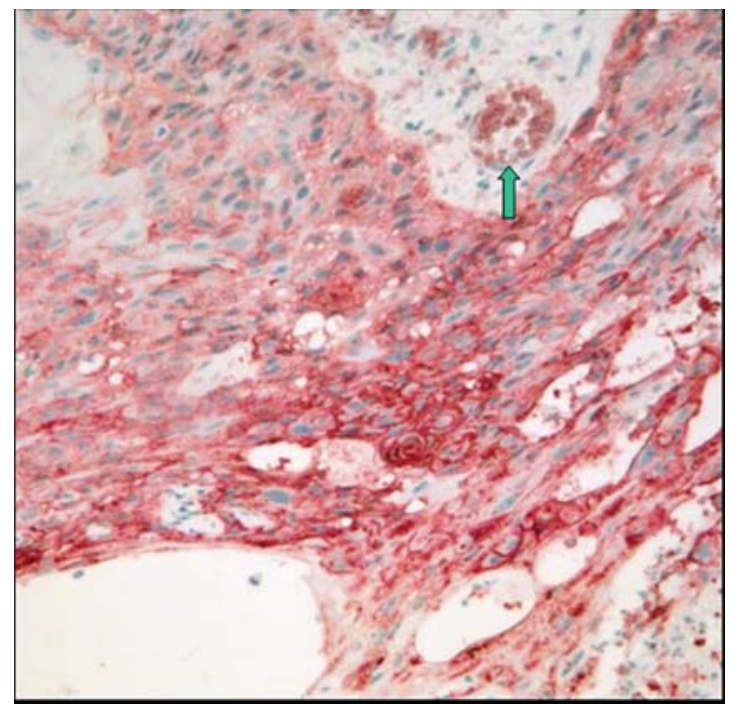

B

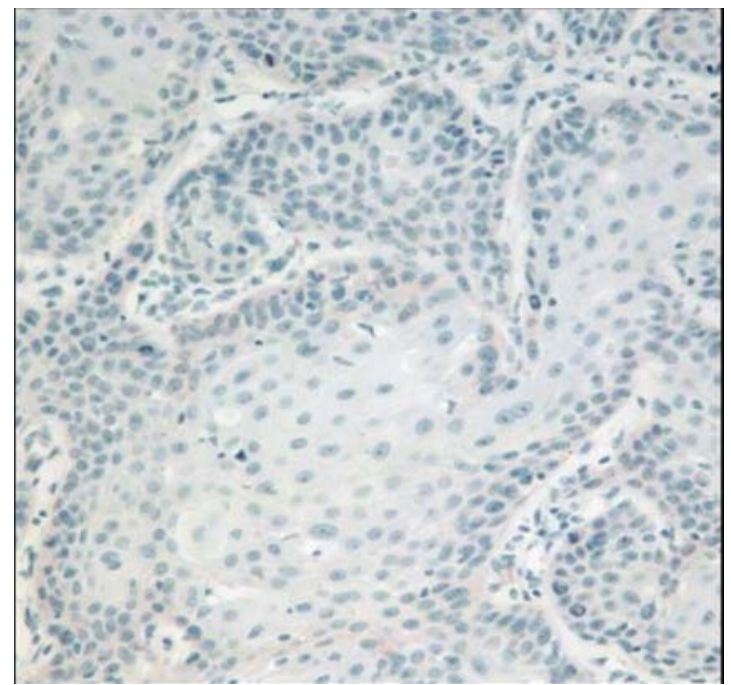

Figure 1. Immunostaining for Glut-1 in OSCC specimens. (A) Patient with poor clinical prognosis. Positive staining for Glut-1 is present in central tumor nests, distal to blood vessels (arrow indicates a vessel; IRS, 12; magnification x100). (B) Patient with good clinical prognosis. No staining for Glut-1 was detected (IRS, 0; magnification $\mathrm{x} 100)$.

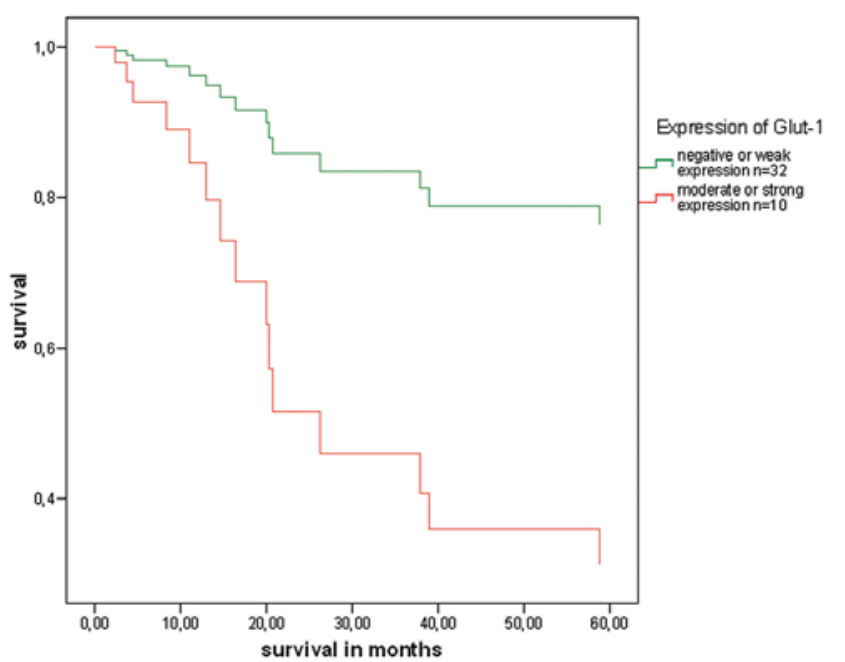

Figure 2. Cox's regression hazard analysis (adj. to tumor size, tumor stage and lymph node stage): Survival time in patients with oral squamous cell carcinoma $(n=42)$ is correlated with expression of Glut- 1 .

investigated the impact of Glut-1 expression on overall survival. In Kaplan-Meier analysis, patients whose tumors showed a moderate to strong expression of Glut-1 had a mean survival time of 25 months compared to 51 months for patients with tumors without or only weak staining for Glut-1 ( $\mathrm{P}=0.001$, Log-rank test).

The five-year survival rates were 24 vs. $74 \%$, respectively (Fig. 2). In multivariate Cox's regression hazard analysis (adj. to tumor size, tumor stage and lymph node stage) a significant 4.9-fold increased risk of tumor-related death could be detected for patients, whose tumors showed a moderate to strong expression for Glut-1 compared to those patients with a negative to weak staining of their tumors $(P=0.015)$. Since the expression of Glut-1 was not significantly correlated to clinical factors relevant to overall survival (tumor size, tumor stage and lymph node stage) it can be considered as an independent prognostic factor for OSCC patients.

Fig. 1 shows an example of high staining intensities for Glut-1 for a patient of poor clinical prognosis and an example of an OSCC poorly stained for Glut-1 from a patient with good clinical prognosis.

\section{Discussion}

It is well known that tumor hypoxia is a characteristic feature of locally advanced solid tumors and can promote tumor progression and resistance to therapy (15-18). Though the effect of hypoxia in malignant tumors of the oral cavity has not been extensively investigated with regard to clinical outcome and prognosis. Tumor hypoxia is an important environmental factor in the control of glucose transporter expression (10). The human transporter, Glut-1, is the major glucose transporter present in human cells $(1,19)$. It has a central role in metabolism with a well-known structure and realizes the transport of D-glucose by facilitated diffusion (20). This transport protein is highly expressed in cells of reticuloendothelial system, cardiac muscle and transformed cells.

The mechanisms of glucose entry into human squamous cell carcinomas remain unclear. Tumor hypoxia is an important environmental factor in the control of glucose transporter expression (21). The role of Glut-1 in carcinogenesis of OSCC is controversially discussed. A significant overexpression of Glut-1 was also noted in other human malignancies. There are studies on the expression of Glut-1 in cervical cancer patients. In recurrent or persistent cervical tumor, tumor size was significantly associated with Glut-1 expression (22). The expression of Glut-1 in laryngeal cancer was also analysed (23). Other reports include the prognostic impact of Glut-1 expression in thyroid (24), ovarian (25), 
esophageal (26), pancreatic (27), prostastic (28), breast (29, 30) and gastrointestinal carcinoma (31).

Glut-1 staining occurs in outer epithelial layers, which may be a function of the differentiation and invasion process. However, Glut-1 staining also exists in central tumor nests, distal to blood vessels, may signify the existence of hypoxiainduced Glut-1 (9). The role of Glut-1 in carcinogenesis of OSCC is controversially discussed. Baer and colleagues reported that Glut-1 is overexpressed in associated squamous epithelial layers suggesting that Glut-1 is ineffective as a prognostic indicator (23). In this study, patients whose tumors carried a moderate to strong expression of Glut-1 had a 4.9-fold increased risk of tumor-related death compared to patients with low or undetectable levels of Glut-1 expression in their tumors. There are two reports in the literature, that described a significant correlation between Glut-1 expression and prognosis of OSCC patients $(9,22)$. Only one study has shown in multivariate Cox's regression hazard analysis that the expression of Glut-1 is a significant prognostic factor. Kunkel et al considered strong expression vs. low expression of Glut-1 and found a 2.65-fold increased risk of tumor-related death for the patient group whose tumors showed a strong expression (22). We included patients with a moderate and a strong expression of Glut-1 and found a 4.9-fold increased risk of tumor-related death for these patients. We suggest that a moderate expression should be considered for prognostic statement additionally to the strongly stained tumors.

In summary, our investigations show that the expression of Glut-1 is an independent prognostic factor for OSCC patients. The level of Glut-1 expression is a useful marker that can provide information on tumor aggressiveness and prognosis as well as a marker for screening of high-risk populations for OSCC.

In conclusion, the current prognostic markers, the TumorNodes-Metastases system (TNM) and the histopathological differentiation (grading) are not sufficient for an exact prediction of prognosis in OSCC. Consequently, there is a strong need to develop novel and independent markers. One of the most promising immunomarkers is Glut-1. Glut-1 enables clinicians at an early stage of the tumor disease to analyze its hypoxic status for prognostic statements and to decide on the therapy strategies. We suggest that an investigation of Glut-1 expression should be used at the diagnosis of OSCC already at the beginning.

\section{References}

1. Schimming R: Tumorangiogenese beim Plattenepithelkarzinom der Mundhöhle: Stellenwert, Prognoserelevanz und therapeutische Ansatzpunkte? PhD-Thesis, Albert-Ludwigs-University Freiburg, Germany, 2001.

2. Hausamen JE: Onkologie: Tumorchirurgie. Mund Kiefer GesichtsChir 4: 142-154, 2000.

3. De Vicente JC, Reico OR, Pendas SL and Lopez-Arranz JS: Oral squamous cell carcinoma of the mandibular region: A survival study. Head Neck 23: 536-543, 2001.

4. Lo WL, Kao SY, Chi LY, Wong YK and Chang RC: Outcomes of oral squamuous cell carcinoma in Taiwan after surgical therapy: factors affecting survival. J Oral Maxillofac Surg 61: 751-758, 2003.

5. De Vicente JC, Gutiérrez LMJ, Zapatero AH, Forcelledo MFF, Hernandez-Vallejo G and Arranz JSL: Prognostic significance of p53 expression in oral squamous cell carcinoma without neck node metastases. Head Neck 26: 22-30, 2004.
6. Schliephake H: Prognostic relevance of molecular markers of oral cander - A review. Int J Oral Maxillofac Surg 32: 233-245, 2003.

7. Lothaire P, de Azambuja E, Dequanter D, Lalami Y, Sotiriou C, Andry G, Castro G and Awada A: Molecular markers of head and neck squamous cell carcinoma: promising signs in need of prospective evaluation. Head Neck 28: 256-269, 2006.

8. Uehara M, Sano K, Ikeda H, Sekine J, Iie A, Yokota T, Tobita T, Ohba $\mathrm{S}$ and Inokuchi T: Expression of vascular endothelial growth factor and prognosis in oral squamous cell carcinoma. Oral Oncol 40: 321-325, 2004.

9. Oliver RJ, Woodwards RTM, Sloan P, Thakker NS, Stratford IJ and Airley RE: Prognostic value of facilitative glucose transporter Glut-1 in oral squamous cell carcinomas treated by surgical resection: results of EORTC Translation Research. Eur J Cancer 40: 503-507, 2004.

10. Airley RE, Loncaster J, Raleigh JA, Harris AL, Davidson SE, Hunter RD, West CM and Sratford IJ: Glut-1 and CAIX as intrinsic markers of hypoxia in carcinomas of the cervix: relationship to pimonidazole binding. Int J Cancer 10: 85-91, 2003.

11. Ivanov S, Liao S-Y, Ivanova A, Danilkovitch-Miagkova A, Tzarasova N, Weirich G, Merill MJ, Proescholdt MA, Oldfield EH, Lee J, Zavada J, Waheed A, Sly W, Lerman MI and Stanbridge EJ: Expression of hypoxia-inducible cell-surface transmembran carbonic anhydrases in human cancer. Am J Pathol 158: 905-919, 2001

12. Bussink J, Kaanders JH and van der Kogel AJ: Tumor hypoxia at the micro-regional level: clinical relevance and predictive value of exogenous and endogenous hypoxic cell markers. Radiother Oncol 67: 3-15, 2003.

13. Remmele W: Pathology. 2nd Edition, IV, Springer Heidelberg, New York, p259, 1999.

14. Kaplan EL and Meier P: Nonparametric estimation from incomplete observations. J Am Stat Ass 53: 457-481, 1958.

15. Verma A: Oxygen-sensing in tumors. Curr Opin Clin Nutr Metab Care 9: 366-378, 2006.

16. Vaupel P and Mayer A: Hypoxia in cancer: significance and impact on clinical outcome. Cancer Metastasis Rev 26: 225-239, 2007.

17. Cloherty EK, Hamill S, Levine K and Carruthers A: Sugar transporter regulation by ATP and quarternary structure. Blood Cells Mol Dis 27: 102-107, 2001.

18. Yen TC, See LC, Lai CH, Yah-Huei CW, Ng KK, Ma SY, Lin WJ, Chen JT, Chen WJ, Lai CR and Hsueh S: 18F-FDG uptake in squamous cell carcinoma of the cervix is correlated with glucose transporter 1 expression. J Nucl Med 45: 22-29, 2004.

19. Kato H, Takit J, Miyazaki T, Nakajima M, Fukai Y, Masuda N, Fukuchi M, Manda R, Ojima H, Tsukada K and Kuwano H: Glut-1 glucose transporter expression in esophageal squamous cell carcinoma is associated with tumor aggressiveness. Anticancer Res 22: 2635-2539, 2002.

20. Zuniga FA, Shi G, Haller JF, Rubahkin A, Flynn DR, Iserovich P and Fischbarg J: A three-dimensional model of the human facilitative Glucose transporter Glut-1. J Biol Chem 276: 44970-44975, 2001

21. Harris AL: Hypoxia - a key regulatory factor in human tumour growth. Nat Rev Cancer 2: 38-47, 2002.

22. Kunkel M, Reichert TE, Benz P, Lehr HA, Jeong JH, Wieand S, Bartenstein P, Wagner W and Whiteside TL: Overexpression of Glut-1 and increased glucose metabolism in tumors are associated with a poor prognosis in patients with oral squamous cell carcinoma. Cancer 97: 1015-1024, 2003.

23. Baer S, Casaubon L, Schwartz MR, Marcogliese A and Younes M: Glut 3 expression in biopsy specimens of laryngeal carcinoma is associated with poor survival. Laryngoscope 112: 393-396, 2002.

24. Yasuda M, Ogane N, Hayashi H, Kameda Y, Miyagi Y, Iida T, Mori Y, Tsukinoki K, Minematsu T and Osamura Y: Glucose transporter-1 expression in the thyroid gland. Clinicopathological significance for papillary carcinoma. Oncol Rep 14: 1499-1504, 2005.

25. Ozcan A, Deveci MS, Oztas E, Dede M, Yenen MC, Korgun ET and Gunhan O: Prognostic value of Glut-1 expression in ovarian surface epithelial tumors: a morphometric study. Anal Quant Cytol Histol 27: 181-186, 2005.

26. Kato H, Takita J, Miyazaki T, Nakajima M, Fukai Y, Masuda N, Fukuchi M, Manda R, Ojima H, Tsukada K and Kuwano H: Glut-1 glucose transporter expression in esophageal squamous cell carcinoma is associated with tumor aggressiveness. Anticancer Res 22: 2635-2639, 2002. 
27. Ito H, Duxbury M, Zinner MJ, Ashley SW and Whang EE: Glucose transporter-1 gene expression is associated with pancreatic cancer invasiveness and MMP-2 activity. Surgery 136: 548-556, 2004.

28. Effert P, Beniers AJ, Tamimi Y, Handt S and Jakse G: Expression of glucose transporter 1 (Glut-1) in cell lines and clinical specimens from humane prostate adenocarcinoma. Anticancer Res 24: 3057-3063, 2004.

29. Stackhouse BL, Williams H, Berry P, Russell G, Thompson P, Winter JL and Kute T: Measurement of glut-1-expression using tissue microarrays to determine a rare specific prognostic marker for breast cancer. Breast Cancer Res Treat 93: 247-253, 2005.
30. Brown RS and Wahl RL: Overexpression of Glut-1 Glucose transporter in human breast cancer. An immunohistochemical study. Cancer 72: 2979-2985, 1993.

31. Cooper R, Sarioglu S, Sokmen S, Fuzun M, Kupelioglu A, Valentine H, Gorken IB, Airley R and West C: Glucose transporter-1 (Glut-1): a potential marker of prognosis in rectal carcinoma? Br J Cancer 89: 870-876, 2003. 